\title{
Long-lived nonthermal electron distribution in aluminum excited by femtosecond extreme ultraviolet radiation
}

\author{
Francesco Bisio, ${ }^{1, *}$ Emiliano Principi, ${ }^{2}$ Michele Magnozzi, ${ }^{3}$ Alberto Simoncig, ${ }^{2}$ Erika Giangrisostomi, ${ }^{2}$ \\ Riccardo Mincigrucci, ${ }^{2}$ Luca Pasquali, ${ }^{4,5}$ Claudio Masciovecchio, ${ }^{2}$ Federico Boscherini, ${ }^{5,6}$ and Maurizio Canepa ${ }^{3}$ \\ ${ }^{1}$ CNR-SPIN, C.so Perrone 24, I-16152 Genova, Italy \\ ${ }^{2}$ Elettra-Sincrotrone Trieste S. C. p. A., Strada Statale 14, km 163.5, 34149 Basovizza, Italy \\ ${ }^{3}$ OptMatLab, Dipartimento di Fisica, Università di Genova, via Dodecaneso 33, I-16146 Genova, Italy \\ ${ }^{4}$ Dipartimento di Ingegneria “Enzo Ferrari,” Università di Modena e Reggio Emilia, Via Vivarelli 10, 41125 Modena, Italy \\ ${ }^{5}$ CNR-IOM, Strada Statale 14, Km. 163.5 in AREA Science Park, 34149 Basovizza, Trieste, Italy \\ ${ }^{6}$ Dipartimento di Fisica e Astronomia, Università di Bologna, Viale C. Berti Pichat 6/2, 40127, Bologna, Italy
}

(Received 10 April 2017; revised manuscript received 2 August 2017; published 30 August 2017)

\begin{abstract}
We report a time-resolved study of the relaxation dynamics of Al films excited by ultrashort intense free-electron laser (FEL) extreme ultraviolet pulses. The system response was measured through a pump-probe detection scheme, in which an intense FEL pulse tuned around the $\mathrm{Al} L_{2,3}$ edge $(72.5 \mathrm{eV})$ acted as the pump, while a time-delayed ultrafast pulse probed the near-infrared (NIR) reflectivity of the Al film. Remarkably, following the intense FEL excitation, the reflectivity of the film exhibited no detectable variation for hundreds of femtoseconds. Following this latency time, sizable reflectivity changes were observed. Exploiting recent theoretical calculations of the EUV-excited electron dynamics [N. Medvedev et al., Phys. Rev. Lett. 107, 165003 (2011)], the delayed NIR-reflectivity evolution is interpreted invoking the formation of very-long-living nonthermal hot electron distributions in $\mathrm{Al}$ after exposure to intense EUV pulses. Our data represent the first evidence in the time domain of such an intriguing behavior.
\end{abstract}

DOI: 10.1103/PhysRevB.96.081119

The interaction of intense ultrashort extreme ultraviolet (EUV) radiation with solid matter is currently a topic of great interest in physics, as it can generate exotic states of matter, such as dense plasmas and warm dense matter (WDM) [1-5]. The impulsive formation and decay of WDM and, in general, of highly excited states of matter created by EUV or $\mathrm{x}$-ray pulses are fast dynamic processes that initially involve the photoexcited electron-gas thermalization and energy transfer to the ion lattice.

In the very early stage after the impulsive and intense EUV excitation, exotic electron populations can be generated in solids. Monitoring their evolution can teach about the dynamics of WDM formation and, in general, the evolution of unconventional electron populations and states of matter. In free-electron-laser (FEL) -based experiments, the choice of the detection technique largely determines which stage of the system evolution and which subsystem is preferentially observed. Measurements of the optical transmission of the exciting pulse, for instance, probe the system evolution on a time scale compatible with the duration of the pulse itself [6-8]. X-ray fluorescence [1-3,5,6] or bremsstrahlung measurements [9] provide a snapshot of the system within few tens of femtoseconds after the excitation pulse, yet probe different excited-electron subsystems [10,11]. Monitoring the system evolution at even longer time delays (picoseconds or more) requires pump-probe experiments with ultrafast probes $[11,12]$.

In this Rapid Communication we report a study of the picosecond dynamics in EUV-excited $\mathrm{Al}$ films, performed by means of a pump-probe experiment, in which an ultrashort

\footnotetext{
*francesco.bisio@spin.cnr.it
}

FEL pulse acted as the pump and a time-delayed near-infrared (NIR) pulse probed the optical reflectivity of the excited system. The EUV pulses were generated by the seeded FEL FERMI@Elettra, with central photon wavelengths of 16.9 and $18.1 \mathrm{~nm}$, i.e., photon energies $\hbar \omega$ above and below the $\mathrm{Al}$ $L$-edge energy threshold $\left(E_{L_{2,3}}\right)$, respectively. Above $L_{2,3}$, light-matter interaction is dominated by $\mathrm{Al} 2 p$ photoexcitation, whereas below this threshold, photoelectric processes proceed via valence-band excitation, effectively allowing one to observe the effects of a very different photon penetration depth in Al. The intensity of the reflected probe pulse with $\lambda=780 \mathrm{~nm}$ was measured as a function of the delay $\Delta t$ between the pump and probe pulses. Remarkably, we observed that the NIRreflectivity dynamics lags by several hundred femtoseconds the arrival of the FEL pulse, before exhibiting a sizable evolution. The unprecedented evidence of such a delayed NIR-reflectivity dynamics can be interpreted invoking recent theoretical findings [10] that predict the formation of longliving hot electron distributions in $\mathrm{Al}$ after exposure to intense EUV pulses due to an electron-thermalization bottleneck. Such a relaxation bottleneck is not a prerogative of high-fluence EUV experiments but occurs also in ordinary EUV or x-ray photoemission. Experiments performed in the spectral domain can, however, obtain only very indirect information based on secondary-electron analysis [13], whereas our data represent the first direct evidence in the time domain of such an intriguing behavior.

The experiments were performed at the EIS-TIMEX beamline at FERMI [14]. The sample consisted of Al films, with a thickness of $1 \mu \mathrm{m}$, deposited onto a $\mathrm{LiF}(001)$ single crystal (Crystec Gmbh) under ultrahigh vacuum conditions and then transported to the beamline for the measurements. Spectroscopic ellipsometry data recorded on the films showed 


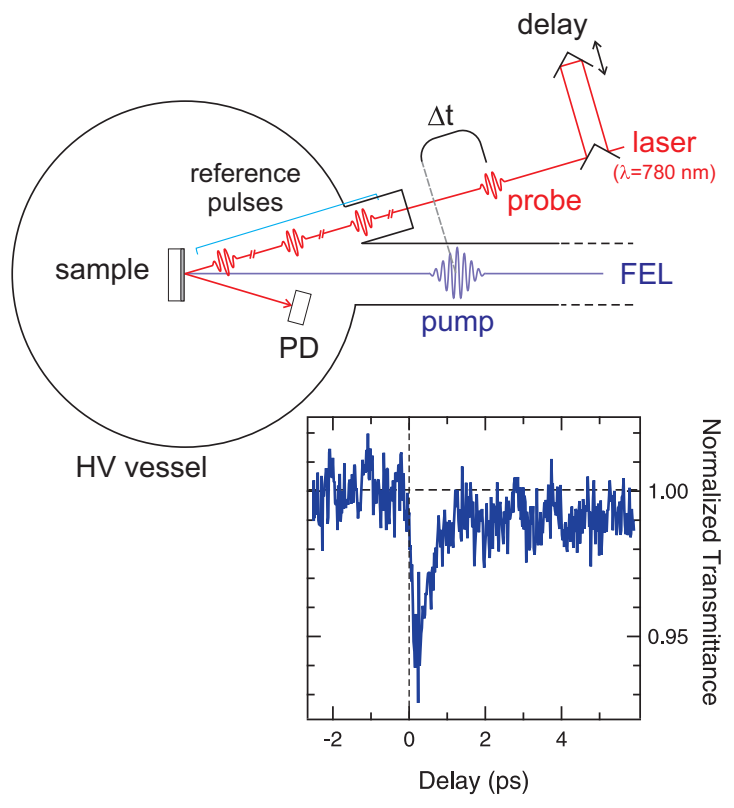

FIG. 1. Top: schematic diagram of the experimental setup at EISTIMEX at FERMI. Bottom: calibration of the zero delay between FEL and laser pulses via the transient optical transmission through a thick $\mathrm{Al}_{2} \mathrm{O}_{3}$ crystal.

the presence of a 3-nm-thick native-oxide layer. The sample was mounted in the experimental chamber of the EIS-TIMEX beamline, held at a pressure of $\approx 10^{-7}$ mbar (see Fig. 1 ). The FEL beam was focused at near-normal incidence (about $0^{\circ}$ ) on the sample to a spot with diameter $\sim 40 \mu \mathrm{m}$. The NIR laser beam $(\lambda=780 \mathrm{~nm})$ passed through a variable-delay stage and was focused onto the Al film also at near-normal incidence (about $10^{\circ}$; see Fig. 1) onto a laser spot with diameter $\sim 30 \mu \mathrm{m}$, in order to ensure to probe a fully excited volume.

The intensity of the reflected laser beam was measured by a photodiode (PD) mounted inside the high-vacuum (HV) chamber. The PD was screened by a thick optical filter in order to cut off undesired background signals originated by FEL and seed laser $(254 \mathrm{~nm})$. The FEL pulse duration $t_{\mathrm{FEL}}$ was estimated to be about 40 fs according to the relation $t_{\mathrm{FEL}} \approx$ $t_{\mathrm{SEED}} / \sqrt{n}$, where $t_{\mathrm{SEED}}=144 \mathrm{fs}$ is the seed pulse duration and $n=14,15$ is the harmonic number selected for the high-gain harmonic generation process [15]. The laser-pulse duration at the sample position was estimated at 96 fs by cross-correlation measurements. In above-threshold excitation, $L$-shell holes get quickly ( $<40 \mathrm{fs}$ ) filled via $L V V$ Auger decay [10], a process dominant with respect to radiative decay for $L$-shell Al [6]. Thus, given the pulse duration, we do not have the time resolution to have direct access to the Auger process but rather observe the evolution of the high-energy electronpopulation tail arising from the Auger decay. The temporal overlap between the laser and the FEL pulses was coarsely set measuring the response of a dedicated in-vacuum radiofrequency cable that generates an electrical pulse with a rise time of about 100 ps when exposed to either the FEL or the laser pulses, and then finely tuned measuring the ultrafast FELinduced optical absorption change in an EUV-excited $\mathrm{Al}_{2} \mathrm{O}_{3}$ single crystal (Fig. 1, bottom). The zero-delay mark has been set in correspondence to the onset of the FEL-induced opacity.
We notice that the time interval between the opacity onset and its peak value is $\approx 200 \mathrm{fs}$, hence the uncertainty in the determination of the absolute delay time $\Delta t$ is safely below $100 \mathrm{fs}$ [16]. It is important to stress that the NIR probe pulse is obtained by splitting the FEL seed pulse prior to frequency tripling, thus guaranteeing sub-10-fs time jitter between the pump and probe pulses [17].

The reflectivity measurements were performed as follows. Three laser pulses were delivered on the sample at the FEL repetition rate $(10 \mathrm{~Hz})$ while preventing FEL lasing, thus serving as reflectivity reference for the unperturbed system $\left(R_{0}\right)$. The very-high reflectivity of $\mathrm{Al}$ in the NIR range ensured a strong signal could be observed. Following the reference pulses, seeded-FEL emission was allowed, and the sample was irradiated with single FEL and laser pulses, separated by the time delay $\Delta t$. The polarization of the FEL beam was circular, in order to obtain from FERMI the highest possible intensities. Since the FEL irradiation led to irreversible sample damage, after each FEL shot the sample was shifted by $120 \mu \mathrm{m}$ to a new position, automatically realigned on the focal plane of the ellipsoidal mirror, and the full sequence was repeated. We point out that irreversible damage was observed for both photon energies employed. For each value of $\Delta t, N$ FEL shots (variable from a few tens to a few hundred), each on a pristine sample position, were recorded, in order to accumulate significant statistics. The whole procedure allowed FEL shots to be fired at a frequency of about $0.5 \mathrm{~Hz}$. The normalized reflectivity $R(\Delta t) / R_{0}$ was calculated as the ratio between the reflected laser intensity measured in correspondence to the FEL shot and the reflected intensity averaged over the three reference laser shots (we notice that for $\Delta t<0$ values, this ratio is unity). The results obtained for each individual shot were then binned according to their delay $\Delta t$ (minimum step: $50 \mathrm{fs}$ ) and the mean $R(\Delta t) / R_{0}$ value corresponding to each delay was extracted.

The data were measured in correspondence to two different central wavelengths of the FEL pulse, $16.9 \mathrm{~nm}$ (photon energy $\hbar \omega=73.3 \mathrm{eV})$ and $18.1 \mathrm{~nm}(\hbar \omega=68.5 \mathrm{eV})$, corresponding to the 15th and 14th harmonics of the seed-laser wavelength and located above and below the Al $L_{2,3}$ edge (typical $\Delta \lambda / \lambda \approx$ $\left.10^{-3}\right)$. Two sets of data were measured, with average pulse energy on the sample of 40 and $60 \mu \mathrm{J}$ (corresponding to a FEL fluence of $\approx 3 \mathrm{~J} / \mathrm{cm}^{2}, \approx 4.5 \mathrm{~J} / \mathrm{cm}^{2}$, respectively), as regulated by means of a combination of filters and a gas-filled attenuation chamber. The FEL intensity at the source was provided by a calibrated ionization chamber and scaled by about $30 \%$ as an effect of the beam transport losses to yield the FEL intensity at the sample. The probe beam was linearly polarized in the horizontal plane.

In Fig. 2 we report the normalized reflectivity $R(\Delta t) / R_{0}$ of the Al film as a function of the delay between the pump and probe pulses. The red symbols represent data collected with $16.9 \mathrm{~nm}$ FEL central wavelength, whereas the blue markers represent data collected with $18.1 \mathrm{~nm}$ central FEL wavelength. The FEL intensity was $40 \mu \mathrm{J} /$ pulse. In the inset, we report a zoom over the zero-delay region for the above-threshold data. In the graphs, data with smaller uncertainty (i.e., that are the result of a larger number of averaged FEL shots) are reported with a darker color hue and larger marker size in order to give them more visual impact. 


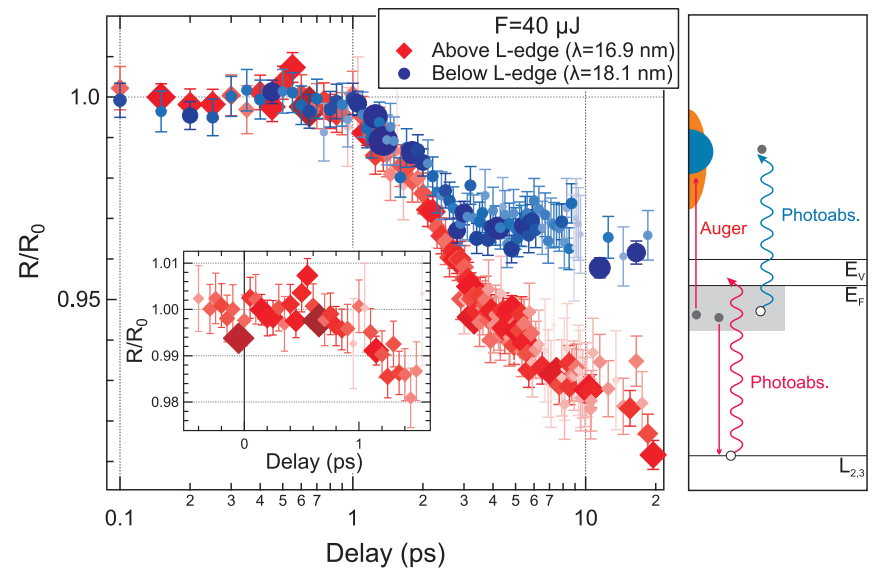

FIG. 2. Left: Normalized reflectivity $R(\Delta t) / R_{0}$ as a function of the pump-probe delay time. Red (blue) markers correspond to data measured for FEL central wavelength of $16.9 \mathrm{~nm}(18.1 \mathrm{~nm})$, i.e., at photon energy above (below) the $\mathrm{Al} L$ edge. The marker size is inversely proportional to the statistical error associated with each data point. Larger markers with darker hue correspond to more reliable data. Inset: Zoom around the zero-delay region for 16.9 $\mathrm{nm}$ radiation at $40 \mu \mathrm{J} /$ pulse. Right: Sketch of above- (red lines) and below-threshold (blue lines) photoexcitation schemes and shortdelay nonthermal populations. Wavy lines represent photoinduced transitions; straight lines represent Auger transitions. The grayshaded area represents the valence band. The orange (blue) areas are a pictorial representation of the high-energy excited-electron populations at very small $\Delta t$.

The most striking observation is that the NIR reflectivity remains nearly constant for about 1 ps after the FEL irradiation before exhibiting any evolution. In the inset, it can be noticed that the experimental points actually start deviating from unity slightly before the picosecond-delay mark, possibly around $\Delta t \approx 600$ fs. After this latency time, $R(\Delta t) / R_{0}$ starts to drop quickly, reaching 0.94 (0.97) for above- (below-) threshold excitation at $\Delta t \approx 3 \mathrm{ps}$ and then decreasing more slowly up to the 20 ps mark. Control measurements at long delays show no recovery of the reflectivity up to $\Delta t \approx 350 \mathrm{ps}$, as indeed expected in the presence of irreversible sample damage.

Figure 3 reports a comparison between the $R(\Delta t) / R_{0}$ curves for above-threshold excitation at $40 \mu \mathrm{J}$ (red markers) and $60 \mu \mathrm{J}$ (open blue markers) excitation energies. The two curves overlap over all the investigated range, within experimental uncertainty.

Before discussing the data, we remark that pump and probe pulses experience different penetration depths $\lambda_{\text {abs }}$ in the Al film. For below-threshold EUV pulses, $\lambda_{\text {abs }}$ in excess of $1 \mu \mathrm{m}$ are indeed predicted [6,19], whereas for above-threshold pulses, $\lambda_{\text {abs }}$ drops to $\approx 50 \mathrm{~nm}$ considering the dielectric function of weakly excited $\mathrm{Al}$ [20], a value extended by $10 \%$ and $17 \%$ by saturable-absorption effects for $3 \mathrm{~J} / \mathrm{cm}^{2}$, and $4.5 \mathrm{~J} / \mathrm{cm}^{2}$, respectively, corresponding to a peak power density in the near-surface region of approximately $10^{19} \mathrm{~W} / \mathrm{cm}^{3}$ [6]. NIR radiation probes instead the few outermost nanometers of the metallic film $\left(\lambda_{\text {abs }}<10 \mathrm{~nm}\right)$. Thus, while the FEL energy may be deposited up to a significant depth within the film, only the near-surface region is probed by the NIR pulse.

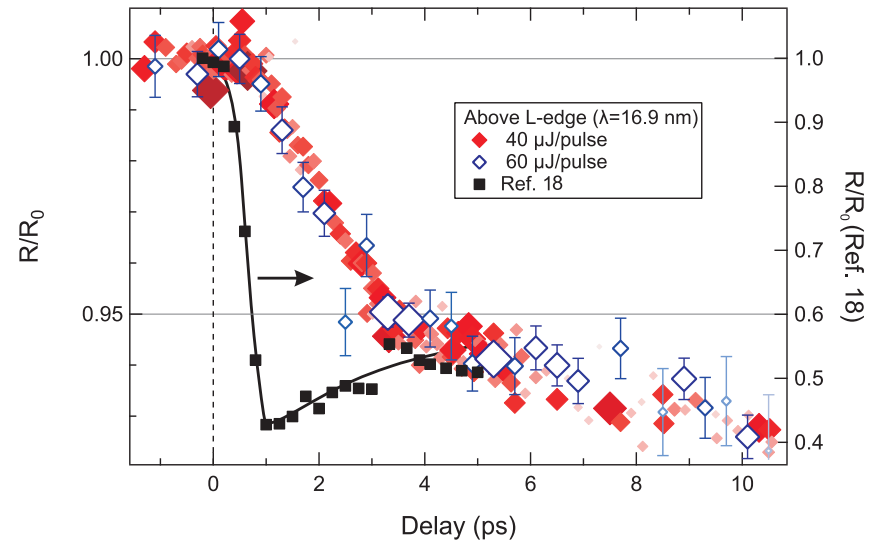

FIG. 3. Normalized reflectivity $R(\Delta t) / R_{0}$ as a function of the pump-probe delay time for FEL central wavelength of $16.9 \mathrm{~nm}$. Red (open blue) markers correspond to data measured with average FEL pulse energy of $40 \mu J(60 \mu J)$. The marker size is inversely proportional to the statistical error associated with each data point. Larger markers correspond to more-reliable data. Black markers: Time-dependent normalized optical reflectivity measured for lowphoton-energy excitation experiments (Ref. [18]). Notice the different reflectivity scale between our data and those of Ref. [18].

Looking at the data, we can identify two distinct ranges of interest: the subpicosecond $\Delta t$ range and the long-delay dynamics. In this Rapid Communication, we will chiefly focus on the former interval. In the subpicosecond range, the main point of interest is represented by the lagged dynamics of the optical reflectivity with respect to the arrival of the FEL pulse. Such a latency time, extremely long considering the typical time scales of electrodynamics in metals, is unmatched in corresponding pump-probe reflectivity experiments employing high-intensity yet low-photon-energy pump-probe combinations, where sudden drops of Al reflectivity are witnessed upon the arrival of the pump [18,21,22]. For the sake of comparison, the experimental data from one such experiment [18] are reported in Fig. 3 as the black markers. The different temporal onset of the reflectivity drop is striking (notice that the reflectivity scale is not the same as our data). Recent theoretical work helps formulate a model for this intriguing behavior.

The absorption of EUV radiation with energy just above $E_{L_{2,3}}$ in Al mainly proceeds by $\mathrm{Al} 2 p$ photoexcitation into the valence band $[6,10]$, whereas radiation with $\hbar \omega<E_{L_{2,3}}$ essentially promotes valence-band electrons to high-lying free-electron-like states far above the Fermi level $E_{F}$ (see scheme in Fig. 2, right).

$L$-shell holes get quickly ( $<40 \mathrm{fs}$ ) filled via $L V V$ Auger decay [10], so that, immediately after the characteristic time for $L$-shell hole recombination has elapsed, the electron population is given by the superposition of a weakly perturbed cold Fermi distribution and a low-density high-energy tail around $65 \mathrm{eV}$ above $E_{F}$ [10], whose energy distribution depends on the energetics of the $L V V$ Auger decay (relative energy difference between $E_{L_{2,3}}$ and valence band, and valence-band energy width).

The populations induced by above- and below-threshold excitation differ in the average energy of the high-lying electron tail, and in the excited-electron density, which is 
expected to be lower in the below-threshold case due to the larger photon penetration depth. The relaxation of such a population initially proceeds by electron-gas thermalization, as the large energy stored within the high-energy population peak is distributed among valence-band electrons via collisions between the high-energy electrons and the Fermi bath. Theoretical prediction for EUV-excited Al fix the electron-gas thermalization time in $\mathrm{Al}$ at a few hundred femtoseconds [10]. This long time, for electronic standards, is essentially due to a thermalization bottleneck, as the large pulse energy is stored in a relatively small number of highly excited carriers, and requires several inelastic collision events to be effectively spread to the Fermi sea.

According to this scheme, we propose that the reflectivity lagging is a direct consequence of the extremely long thermalization time of EUV-excited electrons: indeed, since the Fermi occupation function of the high-energy electron tail, at any time delay, is well below $10^{-2}$ [10], such electrons, despite having a large energy stored, give a very marginal contribution to the overall optical reflectivity, for which large values of joint density of states are required to provide a sizable contribution. $R(\Delta t)$ begins therefore to change only when the energy deposited by the FEL has effectively led to electron-gas thermalization, a process that occurs several hundred fs after the pulse due to the peculiarity of the EUV excitation [10].

The reflectivity plateau is extremely similar, within experimental uncertainty, for above- and below-threshold excitation. This is because the characteristic time scale for Auger recombination (40 fs) is too small to appreciate any difference due to the different relaxation pathways in the two cases, and because the scattering rate of energetic electrons with the Fermi bath does not depend upon their density, but just on their energy.

Having discussed the subpicosecond range, we briefly address the long-delay dynamics. After the electron-gas thermalization has occurred the energy is transferred to the lattice, with an expected electron-phonon coupling time over a picosecond [23]. The strong heating that ensues leads to hydrodynamic expansion and material sublimation, testified by the final irreversible sample damage observed after the experiment. In this delay range, the optical reflectivity follows a monotonically decreasing trend. Although the actual interpretation of the reflectivity curves for $\Delta t>1 \mathrm{ps}$ is not the main focus of this Rapid Communication, some useful indications about the long-delay evolution of the Al film can be deduced comparing our data with the outcome of corresponding high-fluence low-photon-energy experiments employing power densities in the near-surface region between $10^{18}$ and $10^{19} \mathrm{~W} / \mathrm{cm}^{3}$. Reference [18] reports a fast drop (1 ps) and slow recovery (several picoseconds) of reflectivity. References [21,22,24] also report significant drops of reflectivity immediately following the pump $(<1 \mathrm{ps})$, yet without recovery within the first 3 ps. In all cases, the entity of the reflectivity drop is much larger than in our case ( $R$ exhibits $25 \%$ to $80 \%$ drops). The authors of these works account for the behavior observed in terms of a combined heating of electron gas and lattice, material melting [22], and the onset of material rarefaction due to incipient expansion, that preludes to $\mathrm{Al}$ ablation and permanent damage. In general, we can safely infer that the above processes (material heating, rarefaction, and ablation) are most likely all at play in our system for time delays in excess of $1 \mathrm{ps}$, as indeed proved by the permanent damage of the samples after the FEL shot. The measured reflectivity of a FEL-damaged sample is indeed lower than the flat-surface case due to the large scattering within the crater and the possible partial exposure of the substrate. The gradual reflectivity drop that we observe takes place over several picoseconds, a time significantly larger with respect to analogous low-photon-energy experiments. This likely stems from the more even energy distribution throughout the material achieved by FEL irradiation, as opposed to the strong near-surface power dissipation typical of low-photon-energy experiments. The different entity of the reflectivity drops ( $<10 \%$ in our case vs up to $80 \%$ for low-photon energy [21]) has the same origin: indeed, since the penetration depth for visible radiation is typically at least one order of magnitude less than our FEL radiation, it is understandable that the surface region gets more heavily affected by a low-photon-energy pump rather than by FEL irradiation. Therefore, the smaller $\lambda_{\mathrm{abs}}$, the larger the reflectivity variation, and vice versa (see, e.g., the different $\Delta R$ between the above- and below-threshold reflectivity curves in Fig. 2). Conversely, the unexpected similarity between the curves recorded for above-threshold 40 and $60 \mu \mathrm{J}$ pulse energy can be ascribed to the occurrence of saturable absorption in the Al film, which is expected to be tangible for the FEL fluences used in this experiment [6]. In other words, higher above-threshold FEL intensity implies larger penetration depth, hence an effective saturation of the power density deposited by the FEL within the near-surface region, which translates in strongly similar reflectivity curves. Decreasing the FEL intensity below $40 \mu \mathrm{J}$ could reveal the onset of this saturation behavior.

Summarizing, we have reported an experimental investigation of the time-dependent optical reflectivity of Al thin films irradiated with FEL pulses at energies around the $L_{2,3}$ edge. We performed a pump-probe experiment employing the FEL pulse as the pump and a time-delayed near-IR pulse as the probe. We could reliably focus on the short-delay regime thanks to the unique laser-seeded operational mode of FERMI@Elettra, which allows one to minimize the jitter between pump and probe pulses. We observe that, following the irradiation, the dynamics of the optical reflectivity lags by several hundreds of femtoseconds the arrival of the FEL pulse, a behavior at odds with corresponding low-photon-energy pump-probe experiments, where the reflectivity dynamics begins right in correspondence to the arrival of the pump pulse. We suggest that the picosecond delay before the onset of the optical-reflectivity dynamics reflects the different excitation-relaxation pathway that EUV-excited electrons take with respect to low-photonenergy excitation. Indeed, FEL excitation creates a highly energetic yet low-density population of excited electrons that does not macroscopically affect the optical reflectivity until the large energy stored in this low-density population is effectively transferred to the Fermi bath. The thermalization time of the nonequilibrium electron distribution is therefore exceedingly long if compared with typical electron-electron coupling time of free electrons in metals since the concentration of a large amount of energy in a relatively low number of electrons effectively creates a thermalization bottleneck. We point out that the relaxation bottleneck is not a prerogative of high-fluence EUV photoexcitation of core-level electrons 
but occurs in correspondence to any core-level excitation event (i.e., in ordinary photoemission). The high-fluence and ultrashort temporal structure of the FEL excitation merely make it directly observable in the time domain, whereas indirect evidence (secondary emission, linewidth broadening) has to be relied upon in conventional experiments.

Our data corroborate theoretical calculations [10] that predict the occurrence of long-living hot tails of the electron distribution function in $\mathrm{Al}$ excited by an EUV pump above $E_{L_{23} 3}$, but we show that similar effects can be triggered by intense EUV pumps tuned below $E_{L_{2,3}}$. In that case, the hot electron distribution is directly generated by valence electrons excited in the conduction band. Being the maximum energy of hot electrons dictated by the Auger process, hotter nonequilibrium distributions could be obtained at X-ray FEL sources, by exciting deeper core levels in metals.

Financial support from Sincrotrone Elettra SCpA and CNISM (PIK Ex.Pro.Rel.) and from the Ministero dell'Istruzione, dell'Università e della Ricerca (PRIN NEWLI, Grant No. 2015CL3APH) is acknowledged. The authors thank E. Vigo for technical assistance.
[1] B. I. Cho, K. Engelhorn, S. M. Vinko, H.-K. Chung, O. Ciricosta, D. S. Rackstraw, R. W. Falcone, C. R. D. Brown, T. Burian, J. Chalupský, C. Graves, V. Hájková, A. Higginbotham, L. Juha, J. Krzywinski, H. J. Lee, M. Messersmidt, C. Murphy, Y. Ping, N. Rohringer et al., Phys. Rev. Lett. 109, 245003 (2012).

[2] O. Ciricosta, S. M. Vinko, H.-K. Chung, B.-I. Cho, C. R. D. Brown, T. Burian, J. Chalupský, K. Engelhorn, R. W. Falcone, C. Graves, V. Hájková, A. Higginbotham, L. Juha, J. Krzywinski, H. J. Lee, M. Messerschmidt, C. D. Murphy, Y. Ping, D. S. Rackstraw, A. Scherz et al., Phys. Rev. Lett. 109, 065002 (2012).

[3] P. Sperling, E. J. Gamboa, H. J. Lee, H. K. Chung, E. Galtier, Y. Omarbakiyeva, H. Reinholz, G. Röpke, U. Zastrau, J. Hastings, L. B. Fletcher, and S. H. Glenzer, Phys. Rev. Lett. 115, 115001 (2015).

[4] S. M. Vinko, O. Ciricosta, T. R. Preston, D. S. Rackstraw, C. Brown, T. Burian, J. Chalupsk, B. I. Cho, H.-K. Chung, K. Engelhorn, R. W. Falcone, R. Fiokovinini, V. Hjkov, P. A. Heimann, L. Juha, H. J. Lee, R. W. Lee, M. Messerschmidt, B. Nagler, W. Schlotter et al., Nat. Commun. 6, 6397 (2015).

[5] S. M. Vinko, O. Ciricosta, B. I. Cho, K. Engelhorn, H.-K. Chung, C. R. D. Brown, T. Burian, J. Chalupsky, R. W. Falcone, C. Graves, V. Hajkova, A. Higginbotham, L. Juha, J. Krzywinski, H. J. Lee, M. Messerschmidt, C. D. Murphy, Y. Ping, A. Scherz, W. Schlotter et al., Nature (London) 482, 59 (2012).

[6] B. Nagler, U. Zastrau, R. R. Fäustlin, S. M. Vinko, T. Whitcher, A. J. Nelson, R. Sobierajski, J. Krzywinski, J. Chalupský, E. Abreu, S. Bajt, T. Bornath, T. Burian, H. Chapman, J. Cihelka, T. Döppner, S. Düsterer, T. Dzelzainis, M. Fajardo, E. Förster et al., Nat. Phys. 5, 693 (2009).

[7] A. Di Cicco, K. Hatada, E. Giangrisostomi, R. Gunnella, F. Bencivenga, E. Principi, C. Masciovecchio, and A. Filipponi, Phys. Rev. B 90, 220303 (2014).

[8] D. S. Rackstraw, O. Ciricosta, S. M. Vinko, B. Barbrel, T. Burian, J. Chalupský, B. I. Cho, H.-K. Chung, G. L. Dakovski, K. Engelhorn, V. Hájková, P. Heimann, M. Holmes, L. Juha, J. Krzywinski, R. W. Lee, S. Toleikis, J. J. Turner, U. Zastrau, and J. S. Wark, Phys. Rev. Lett. 114, 015003 (2015).

[9] U. Zastrau, C. Fortmann, R. R. Fäustlin, L. F. Cao, T. Döppner, S. Düsterer, S. H. Glenzer, G. Gregori, T. Laarmann, H. J. Lee, A. Przystawik, P. Radcliffe, H. Reinholz, G. Röpke, R. Thiele, J. Tiggesbäumker, N. X. Truong, S. Toleikis, I. Uschmann, A. Wierling, T. Tschentscher, E. Förster, and R. Redmer, Phys. Rev. E 78, 066406 (2008).
[10] N. Medvedev, U. Zastrau, E. Förster, D. O. Gericke, and B. Rethfeld, Phys. Rev. Lett. 107, 165003 (2011).

[11] S. P. Hau-Riege, Phys. Rev. E 87, 053102 (2013).

[12] M. Harmand, R. Coffee, M. R. Bionta, M. Chollet, D. French, D. Zhu, D. M. Fritz, H. T. Lemke, N. Medvedev, B. Ziaja, S. Toleikis, and M. Cammarata, Nat. Photon. 7, 215 (2013).

[13] S. Hüfner, Photoelectron Spectroscopy: Principles and Applications (Springer, Berlin/Heidelberg, 1996).

[14] C. Masciovecchio, A. Battistoni, E. Giangrisostomi, F. Bencivenga, E. Principi, R. Mincigrucci, R. Cucini, A. Gessini, F. D'Amico, R. Borghes, M. Prica, V. Chenda, M. Scarcia, G. Gaio, G. Kurdi, A. Demidovich, M. B. Danailov, A. Di Cicco, A. Filipponi, R. Gunnella et al., J. Synchrotron Radiat. 22, 553 (2015).

[15] E. Allaria, R. Appio, L. Badano, W. A. Barletta, S. Bassanese, S. G. Biedron, A. Borga, E. Busetto, D. Castronovo, P. Cinquegrana, S. Cleva, D. Cocco, M. Cornacchia, P. Craievich, I. Cudin, G. D’Auria, M. Dal Forno, M. B. Danailov, R. De Monte, G. De Ninno et al., Nat. Photon. 6, 699 (2012).

[16] S. M. Durbin, AIP Adv. 2, 042151 (2012).

[17] M. B. Danailov, F. Bencivenga, F. Capotondi, F. Casolari, P. Cinquegrana, A. Demidovich, E. Giangrisostomi, M. P. Kiskinova, G. Kurdi, M. Manfredda, C. Masciovecchio, R. Mincigrucci, I. P. Nikolov, E. Pedersoli, E. Principi, and P. Sigalotti, Opt. Express 22, 12869 (2014).

[18] M. C. Downer, H. Ahn, D. H. Reitze, and X. Y. Wang, Int. J. Thermophys. 14, 361 (1993).

[19] S. M. Vinko, G. Gregori, M. P. Desjarlais, B. Nagler, T. J. Whitcher, R. W. Lee, P. Audebert, and J. S. Wark, High Energy Density Phys. 5, 124 (2009).

[20] E. D. Palik, Handbook of Optical Constants of Solids (Academic, New York, 1985).

[21] K. Widmann, G. Guethlein, M. E. Foord, R. C. Cauble, F. G. Patterson, D. F. Price, F. J. Rogers, P. T. Springer, R. E. Stewart, A. Ng, T. Ao, and A. Forsman, Phys. Plasmas 8, 3869 (2001).

[22] M. Kandyla, T. Shih, and E. Mazur, Phys. Rev. B 75, 214107 (2007).

[23] B. J. Siwick, J. R. Dwyer, R. E. Jordan, and R. J. D. Miller, Science 302, 1382 (2003).

[24] M. E. Povarnitsyn, N. E. Andreev, E. M. Apfelbaum, T. E. Itina, K. V. Khishchenko, O. F. Kostenko, P. R. Levashov, and M. E. Veysman, Appl. Surf. Sci. 258, 9480 (2012). 